\title{
Forkhead transcription factor 1 inhibits endometrial cancer cell proliferation via sterol regulatory element-binding protein 1
}

\author{
YIFANG ZHANG $^{1,2}$, LILI ZHANG $^{3}$, HENGZI SUN ${ }^{1}$, QINGTAO LV ${ }^{4}$, CHUNPING QIU $^{1}$, \\ XIAOXIA CHE $^{1}$, ZHIMING LIU ${ }^{1}$ and JIE JIANG ${ }^{1}$ \\ ${ }^{1}$ Department of Obstetrics and Gynecology, Qilu Hospital, Shandong University, Jinan, Shandong 250012; \\ ${ }^{2}$ Department of Obstetrics and Gynecology, Affiliated Hospital of Taishan Medical University, Tai'an, Shandong 271000; \\ ${ }^{3}$ Department of Ultrasonography, Jinan Central Hospital Affiliated to Shandong University, Jinan, Shandong 250012; \\ ${ }^{4}$ Department of Pharmaceutical Chemistry, Shandong University of Traditional Chinese Medicine, \\ Jinan, Shandong 250012, P.R. China
}

Received June 11, 2015; Accepted October 4, 2016

DOI: $10.3892 / \mathrm{ol} .2016 .5480$

\begin{abstract}
The morbidity and mortality associated with endometrial cancer (EC) has increased in recent years. Regarded as a tumor suppressor, forkhead transcription factor 1 (FOXO1) has various biological activities and participates in cell cycle progression, apoptosis and differentiation. Notably, FOXO1 also functions in the regulation of lipogenesis and energy metabolism. Lipogenesis is a feature of cancer and is upregulated in EC. Sterol regulatory element-binding protein 1 (SREBP1) is a transcription factor that is also able to regulate lipogenesis. Increased expression of SREBP1 is directly correlated with malignant transformation of tumors. A previous study demonstrated that SREBP1 was highly expressed in EC and directly resulted in tumorigenesis. However, the association between FOXO1 and SREBP1 in EC is not clear. In the present study, lentiviruses overexpressing FOXO1 were used in cell transfection and transduction. Cell viability assays demonstrated that the overexpression of FOXO1 was able to suppress cell proliferation significantly in Ishikawa and AN3 CA cell lines. In addition, FOXO1 overexpression significantly inhibited cell migration and invasion ability in vitro. In xenograft models, overexpression of FOXO1 suppressed cell tumorigenesis, and western blot analysis demonstrated that SREBP1 expression was markedly reduced in the FOXO1-overexpressing cells. It may therefore be concluded that FOXO1 is able to inhibit the proliferative capacity of cells in vitro and in vivo, in addition to the migratory and invasive capacities in vitro by directly targeting SREBP1.
\end{abstract}

Correspondence to: Professor Jie Jiang, Department of Obstetrics and Gynecology, Qilu Hospital, Shandong University, 107 Wenhua West Road, Jinan, Shandong 250012, P.R. China

E-mail: qljiangjie@sdu.edu.cn

Key words: forkhead transcription factor 1, sterol regulatory element-binding protein 1 , endometrial cancer

\section{Introduction}

Endometrial cancer (EC) has the highest incidence rate of all malignant tumors of the female genital system in the United States $(1,2)$. EC-associated morbidity and mortality has increased in recent years and its incidence is slightly below that of breast, lung and bronchus, and colorectal cancer. According to the American Cancer Society, in 2016 there were 60,050 newly expected cases and 10,470 mortalities associated with EC in the United States (2). Despite treatment options including surgery, radiotherapy and chemotherapy, the prognosis of poorly-differentiated EC is poor (3). Fatty acid and cholesterol synthesis is important in the growth of cancer cells, with ectopic lipid metabolism leading to tumorigenesis (4). Lipogenesis is usually upregulated and obesity occurs in $40 \%$ of all EC cases (5).

FOXO1, which belongs to the FOX transcription factor family, is typically regarded as a tumor suppressor and lies downstream in the phosphoinositide 3-kinase (PI3K)/Akt signaling pathway. This molecule performs various biological activities and participates in energy metabolism $(6,7)$, cell-cycle progression (8), apoptosis, cellular differentiation $(9,10)$, wound healing and stress response (11). Through upregulation of adipose triglyceride lipase and lysosomal acid lipase levels, FOXO1 is able to enhance fat catabolism (12). FOXO1 is directly targeted by insulin and highly expressed in insulin-sensitive tissues (7). Insulin inhibits the action of FOXO1 by binding insulin growth factor (IGF)-1 and FOXO1 receptor, which subsequently activates certain intracellular kinases involved in the PI3K/Akt pathway. Activation of this signaling pathway results in FOXO1 phosphorylation, which reduces FOXO1 nuclear translocation, thereby inhibiting its transcriptional function (12). Previous research has identified that dysregulation of the PI3K/Akt pathway is characteristic of EC (13). Immunohistochemical (IHC) and RT-PCR studies from Ward et al (14) and Goto et al (15) demonstrated that FOXO1 exhibited lower expression levels in EC samples compared with normal endometrium tissue. Loss of FOXO1 expression promotes uncontrolled EC cell proliferation. 
Sterol regulatory element-binding proteins (SREBPs) regulate the expression of lipogenic genes and are members of the basic helix-loop-helix leucine zipper family (3). The family has three different isoforms: SREBP1a, SREBP1c and SRBEP2 (3). Each isoforms has different effects; SREBP1 is the primary SREBP and it selectively regulates intracellular lipid homeostasis by controlling the synthesis of fatty acids and triglycerides (16). High expression of SREBP1 is directly correlated with tumorigenesis in several forms of cancer, including prostate, breast and pancreatic cancer (17-19). SREBP1 is also a target of insulin; insulin is able to activate transcription of the gene encoding SREBP1 by increasing the activity of liver X receptors (16). Using IHC staining, reverse transcription-quantitative polymerase chain reaction (RT-qPCR) and western blotting, a previous study observed a significant increase in SREBP1 protein expression in EC samples and in poorly-differentiated EC cells (3). Similarly, in vitro and in vivo research has demonstrated that SREBP1-knockdown is able to reduce proliferation and induce apoptosis in EC cells (3).

Despite the importance of FOXO1 and SREBP1 in EC, they are also important in lipogenesis and may be regulated by insulin. However, the association between FOXO1 and SREBP1 in EC remains unclear. Therefore, the present study aimed to elucidate this association.

\section{Materials and methods}

Cell lines and reagents. A total of six human EC cells (Ishikawa, AN3 CA, HEC-1-A, SPEC-2, RL95-2 and KLE) were purchased from the American Type Culture Collection (ATCC; Rockville, MD, US). Fetal bovine serum (FBS), Dulbecco's modified Eagle's medium (DMEM)/high glucose medium, minimal essential medium (MEM), McCoy's 5A medium and DMEM/F12 medium were purchased from Gibco, Thermo Fisher Scientific, Inc. (Waltham, MA, USA). Anti-FOXO1 antibodies (no. ab39670) were purchased from Abcam (Cambridge, UK), anti-SREBP1 antibodies (H-160; no. sc-8984) from Santa Cruz Biotechnology, Inc. (Dallas, TX, USA) and anti-GAPDH antibodies (no. G9545) from Sigma-Aldrich; Merck Millipore (Darmstadt, Germany).

Cell culture. All cells were incubated at $37^{\circ} \mathrm{C}$ in $5 \% \mathrm{CO}_{2}$. The Ishikawa and AN3 CA cells were cultured in DMEM/high glucose medium, the RL95-2 and KLE cells in DMEM/F12 medium, the SPEC-2 cells in MEM and the HEC-1-A cells in McCoy's 5A medium. All cell culture media were supplemented with FBS at a concentration of $10 \%$.

Cell transfection and transduction. Lentiviruses expressing green fluorescent (GFP)-tagged proteins with a human FOXO1 overexpression vector (group named as LV-FOXO1) and lentiviruses with a control vector (group named as LV-CON) were constructed and prepared for transfection by GeneChem Co., Ltd. (Shanghai, China). The FOXO1 low-expression cell lines [Ishikawa (well-differentiated EC cells) and AN3 CA (poorly-differentiated EC cells)] were screened for transfection according to western blot analysis. Prior to transduction, cells were incubated on 6-well plates for $24 \mathrm{~h}$ until adherence. Lentiviruses at multiplicity of infection
(MOI) 50 were transduced into cells. At $10 \mathrm{~h}$ post-transduction, the culture medium was replaced to normal medium. A total of $72 \mathrm{~h}$ were required for stable cell transfection. The whole transduction was performed in accordance with the manufacturer's protocol (GeneChem Co., Ltd.). After $72 \mathrm{~h}$, a fluorescence microscope was used to observe GFP-positive cells and western blotting was performed to determine the transduction efficiency.

Western blot analysis. Six types of human EC cells (Ishikawa, AN3 CA, HEC-1-A, SPEC-2, RL95-2 and KLE) were washed with 1X PBS, harvested and lysed with radio immunoprecipitation assay buffer (RIPA buffer, 1\% NP40, 1X PBS, $0.1 \%$ SDS, $5 \mathrm{mM}$ EDTA, $1 \mathrm{mM}$ sodium orthovanadate and $0.5 \%$ sodium deoxycholate) containing phenylmethanesulfonyl fluoride (dilution, 1:100) as a protease inhibitor. The mixture was placed on ice for $30 \mathrm{~min}$ for complete lyse, and was subsequently centrifuged at $13,800 \mathrm{x} g$ for $15 \mathrm{~min}$ at $4^{\circ} \mathrm{C}$. The suspensions were carefully collected and tested for protein concentration using a BCA Protein assay kit (no. p0010; Beyotime Institute of Biotechnology, Haimen, China) [containing reagent $\mathrm{A}$, reagent $\mathrm{B}$ and bovine serum albumin (BSA); reagent A contained sodium carbonate, sodium bicarbonate, bicinchoninic acid and sodium tartrate in $0.1 \mathrm{M}$ sodium hydroxide; reagent $\mathrm{B}$ contained $4 \%$ cupric sulfate; BSA at $2.0 \mathrm{mg} / \mathrm{ml}$ in $0.9 \%$ saline and $0.05 \%$ sodium azide]. Proteins were resolved with SDS-PAGE loading buffer and $30 \mu \mathrm{g}$ each sample was transferred to polyvinylidene fluoride membranes (EMD Millipore, Billerica, MA, USA). The membranes were blocked with non-fat milk (5\%) for $2 \mathrm{~h}$ at room temperature and then incubated with the primary antibodies against FOXO1 (dilution, 1:500), SREBP1 (dilution, 1:1,000) and GAPDH (dilution, 1:1,000) at $4^{\circ} \mathrm{C}$ overnight. Next, the membranes were washed with TBST, and incubated with horseradish peroxidase-coupled rabbit IgG secondary antibody (dilution, 1:5,000; no. 074-1506; Kirkegaard \& Perry Laboratories, Inc., Gaithersburg, MD, USA) for $2 \mathrm{~h}$ at room temperature. Membranes were washed with TBST and analyzed using ImagemQuant ${ }^{\mathrm{TM}}$ LAS 4000 with enhanced chemiluminescence. The protein signals were analyzed with ImageJ software (https://imagej.nih.gov/ij/) and protein levels were compared with those of GAPDH.

Cell proliferation and clonogenic assay. MTT assay was used to assess cell proliferation. Ishikawa (LV-FOXO1 and LV-CON) and AN3 CA (LV-FOXO1 and LV-CON) were seeded in 96-well plates at 4,000 and 3,000 cells/well respectively, and attached overnight. Cells were subsequently incubated at $37^{\circ} \mathrm{C}$ for $1-5$ days, and $20 \mu 15 \mathrm{mg} / \mathrm{ml}$ MTT was added each day to each well at the specified time. Following further incubation for $4 \mathrm{~h}$ at $37^{\circ} \mathrm{C}$, the supernatants were carefully discharged and replaced with $100 \mu$ l dimethyl sulfoxide. Infinite ${ }^{\circledR} 200$ PRO NanoQuant (Tecan Group Ltd., Männedorf, Switzerland) were used to read the absorbance values at $570 \mathrm{~nm}$. To determine clonogenic ability, 400 cells of each group were allowed to grow for 14 days on 6-well plates to form colonies. When distinguished by the naked eye, crystal violet (2\%,w/v; Sigma-Aldrich; Merck Millipore) were used to stain colonies of clone formation and clone numbers were subsequently counted under an inverted microscope. 
A

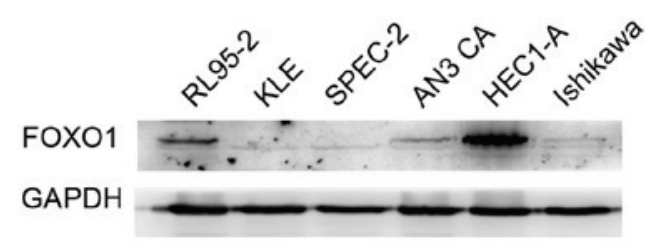

C
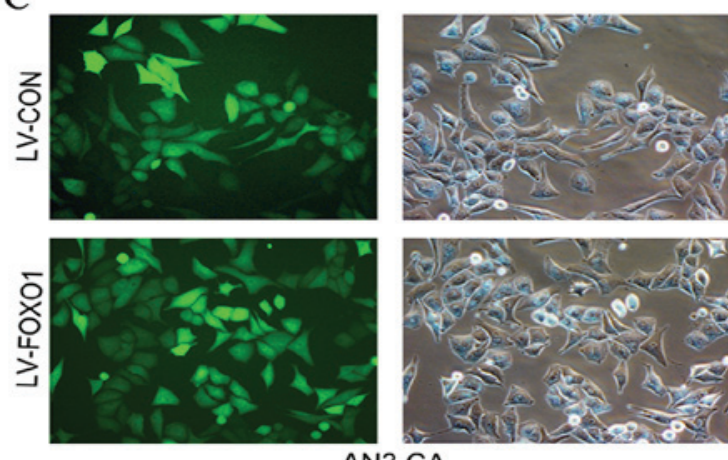

B

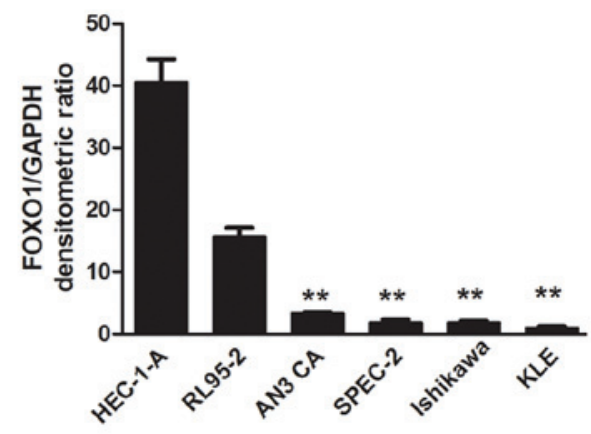

D

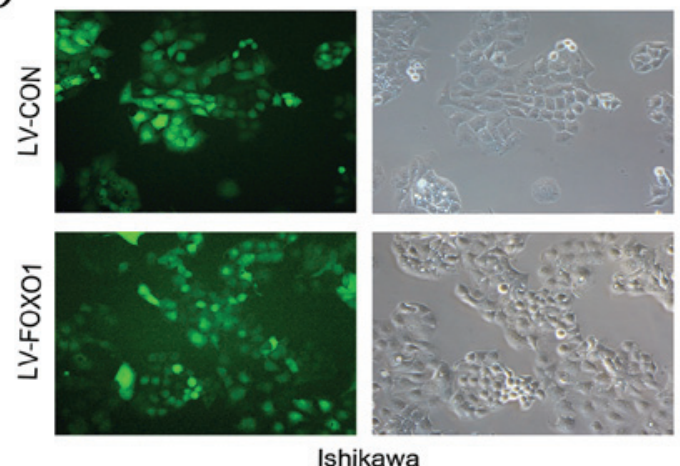

Figure 1. Expression of FOXO1 in human endometrial cancer cells and overexpression of FOXO1 via lentiviruses. (A) Relative FOXO1 expression levels in six different human endometrial cancer cell lines were examined by western blotting and (B) were subsequently quantified. ${ }^{* *} \mathrm{P}<0.01$ vs. FOXO1 expression in HEC-1-A cells. (C) AN3 CA (poorly-differentiated endometrial cancer) and (D) Ishikawa (well-differentiated endometrial cancer) cells were selected and transduced with a lentivirus (expressing GFP-positive green fluorescent protein) containing a FOXO1 overexpression vector (LV-FOXO1 group) and a control vector (LV-CON group). GFP-positive cells were observed (left lane by fluorescence microscope, right lane by inverted microscope). FOXO1, forkhead transcription factor 1; GFP, green fluorescent protein.

Migration and invasion assay. Transwell systems with polycarbonate membranes (24-well, $8 \mathrm{~mm}$ size pore; Costar; Corning Incorporated, Corning, NY, USA) were used to perform migration and invasion assay. Matrigel (BD Biosciences, San Jose, CA, US) was also used to coat the membranes in the invasion assay. A total of $200 \mu 1$ medium free from FBS with $1.5 \times 10^{5}$ cells were added to the upper well, and $700 \mu 1$ medium with $20 \%$ FBS was added to the lower chamber. The cells were incubated for $24 \mathrm{~h}$ for the migration assay and $48 \mathrm{~h}$ for the invasion assay. Cells that had adhered to the lower well were stained with crystal violet and counted under an inverted microscope for six random visual fields.

In vivo tumorigenesis. Two groups of stable transfected AN3 CA cells (LV-FOXO1 and LV-CON) were used to perform in vivo tumorigenesis. Once cells had grown to $70-80 \%$ density, they were digested and counted. A total of $8 \times 10^{6}$ cells from each group were suspended in $200 \mu \mathrm{l}$ PBS (mixture with Matrigel at 3:1). The cells were subsequently injected into the subcutaneous flank of 4-5-week-old BALB/c-nu/nu female mice (raised in a specific pathogen-free laboratory; 18-20 g; 7 mice/group). Following injection, the diameters of the transplanted tumors [length (L) and width (W)] were measured every 4 days using a slide caliper. Tumor volume was calculated as $\left(\mathrm{Lx} \mathrm{W}^{2}\right) / 2$. At day 28 post-injection, the mice were sacrificed under anesthesia, and the tumors were separated, collected and weighed. The Animal Care and Use Committee of Shandong University (Jinan, China) approved all animal experiments.
Statistical analysis. SPSS v17.0 (SPSS, Inc., Chicago, IL, USA) was used for statistical analysis. Data are presented as the mean \pm standard deviation, and each experiment was repeated three times. Student's two-tailed t-test was used to determine statistical significance of two groups, and $\mathrm{P}<0.05$ was considered to indicate a statistically significant difference.

\section{Results}

FOXO1 overexpression suppresses Ishikawa and AN3 CA cell proliferation and colonigenic ability in vitro. FOXO1 expression was analyzed in six different human EC cells (Ishikawa, AN3 CA, HEC-1-A, SPEC-2, RL95-2 and KLE). From western blot analysis, it was observed that the expression of FOXO1 in the AN3 CA, SPEC-2, Ishikawa and KLE cells was lower than that observed in the HEC-1-A and RL95-2 cells (Fig. 1A and $\mathrm{B}$ ). Thus, the two differentiated cell lines (Ishikawa and AN3 CA) were selected for further experiments. Following transfection, GFP-positive cells accounted for $>90 \%$ of the total cells observed by fluorescence microscopy in the AN3 CA (Fig. 1C) and Ishikawa (Fig. 1D) cells. This certified that transfection was successful. To further detect the efficiency of transduction, western blotting was performed. The results demonstrated a significant increase in the expression of FOXO1 protein in the LV-FOXO1 group compared with the LV-CON group for both the Ishikawa $(\mathrm{P}<0.01$; Fig. $2 \mathrm{~A}$ and $\mathrm{B})$ and AN3 CA cells ( $\mathrm{P}<0.05$; Fig. $2 \mathrm{C}$ and $\mathrm{D})$.

A cell viability assay was performed using MTT to examine the effect of FOXO1 on cell growth. The results demonstrated 

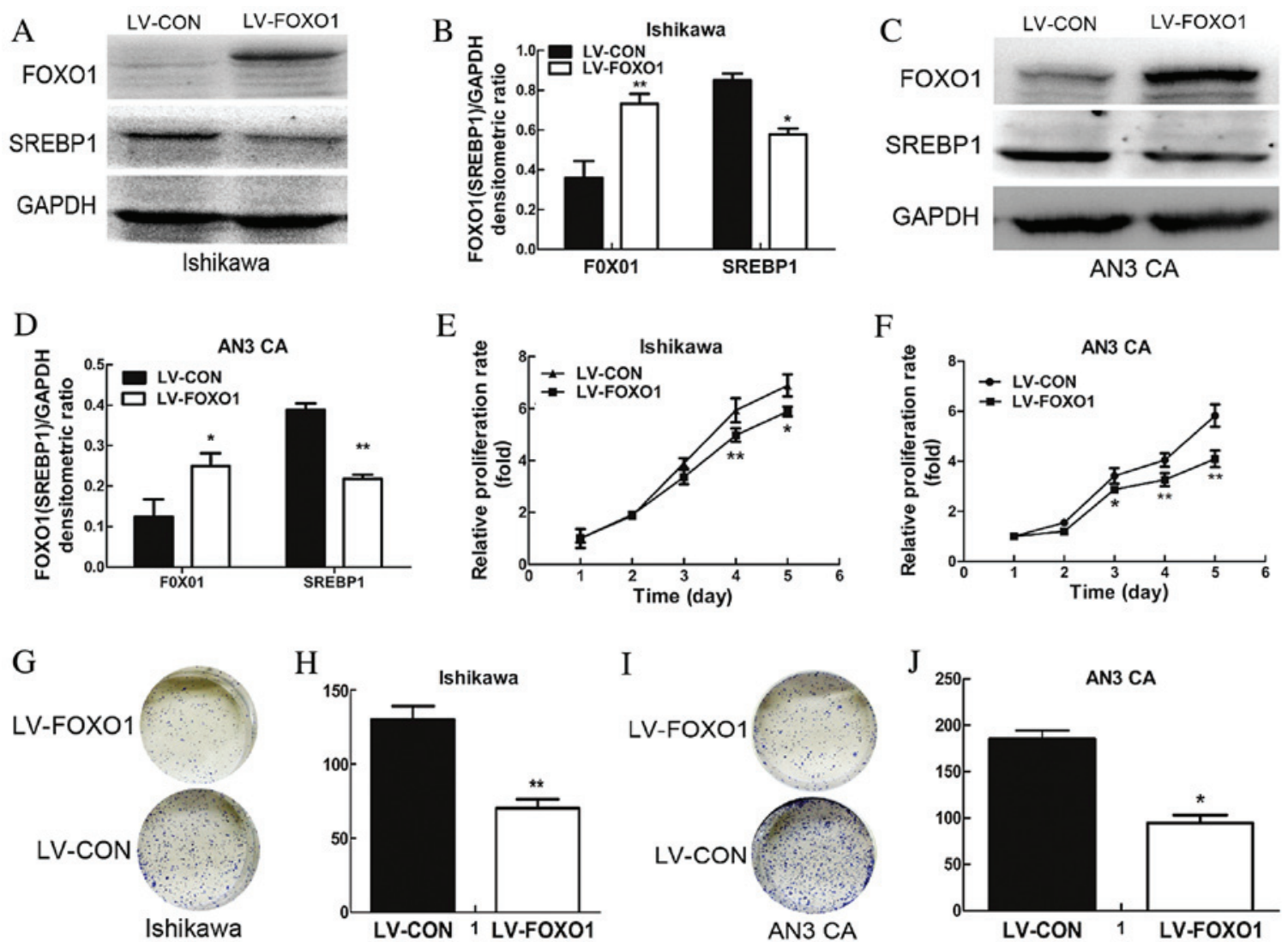

Figure 2. Stable overexpression of FOXO1 suppresses cell proliferation and colonigenic ability in vitro, and FOXO1 inhibited cell migration and invasion by targeting SREBP1. Expression of FOXO1 and SREBP1 of LV-FOXO1 group and LV-CON group in (A and B) Ishikawa and (C and D) AN3 CA cells was validated by western blot analysis. GAPDH serves as a protein control. Effect of FOXO1 overexpression on the proliferation of (E) Ishikawa and (F) AN3 CA cells examined by MTT assay. Effect of FOXO1 overexpression on the colonigenic ability of (G and H) Ishikawa and (I and J) AN3 CA cells. "P<0.05 and ${ }^{* *} \mathrm{P}<0.01$ vs. the LV-CON group. FOXO1, forkhead transcription factor 1; SREBP1, sterol regulatory element-binding protein 1.

that FOXO1 overexpression was able to significantly suppress proliferation of the Ishikawa (day 4, P=0.004 and day 5, $\mathrm{P}=0.034$; Fig. 2E) and AN3 CA cells (day 3, $\mathrm{P}=0.016$, day 4, $\mathrm{P}=0.006$ and day $5, \mathrm{P}=0.001$; Fig. $2 \mathrm{~F}$ ) when compared with the LV-CON group. To further investigate this, colonigenic assay was performed to evaluate the oncogenic potential of FOXO1. It was observed that the colonigenic ability of the LV-FOXO1 group was significantly reduced in the Ishikawa $(\mathrm{P}=0.005$; Fig. 2G and $\mathrm{H})$ and $\mathrm{AN} 3 \mathrm{CA}(\mathrm{P}=0.013$; Fig. 2I and J).

FOXO1 overexpression suppresses Ishikawa and AN3 CA cell migration and invasion in vitro. In order to observe the potential impact of FOXO1 overexpression on the migratory and invasive ability of Ishikawa and AN3 CA cells, a Transwell assay was performed. The results demonstrated that the migratory abilities of the LV-FOXO1 group were significantly inhibited in the Ishikawa $(\mathrm{P}=0.034)$ and $\mathrm{AN} 3 \mathrm{CA}$ $(\mathrm{P}=0.029$; Fig. $3 \mathrm{~A}$ and $\mathrm{B})$ cells, and the invasive abilities of the LV-FOXO1 group was also significantly inhibited in the Ishikawa $(\mathrm{P}=0.019)$ and $\mathrm{AN} 3 \mathrm{CA}$ cells compared with the LV-CON group ( $\mathrm{P}=0.003$; Fig. $3 \mathrm{C}$ and $\mathrm{D})$.

FOXO1 suppresses AN3 CA cell tumorigenesis in a xenograft model. FOXO1 overexpression suppressed the proliferation, and colonigenic abilities of AN3 CA cells in vivo. To investigate the role of FOXO1 expression in cell proliferation, an in vivo experiment was constructed. A xenograft model of human EC was used. Stably transfected AN3 CA (LV-CON) were injected subcutaneously into the flank of nude mice and day 28 post-injection tumors were separated (Fig. 4A and B), and stably transfected AN3 CA (LV-FOXO1) were injected into the flank of nude mice and then tumors were separated (Fig. 4B and C). The results demonstrated that LV-FOXO1 group compared with LV-CON group FOXO1 evidently inhibited tumor formation and growth (tumor size at day 12, day 16, day 20, day 24 and day 28 post-injection, $\mathrm{P}=0.00$; Fig. 4D; tumor weight, $\mathrm{P}=0.00$; Fig. 4E).

FOXO1 inhibits Ishikawa and AN3 CA cell migration and invasion by targeting SREBP1. FOXO1 and SREBP1 are crucial in lipid metabolism $(7,19)$. The present study speculated that FOXO1 functions though cross-talk to SREBP1, therefore, western blot analysis was performed to investigate the association between them. It was concluded that SREBP1 protein expression was markedly decreased in the LV-FOXO1 group in the Ishikawa $(\mathrm{P}=0.016$; Fig. $2 \mathrm{~A}$ and $\mathrm{B})$ and $\mathrm{AN} 3$ CA ( $\mathrm{P}=0.005$; Fig. 2C and $\mathrm{D})$. Therefore, overexpression of FOXO1 is able to downregulate the expression of SREBP1.

\section{Discussion}

As a member of the forkhead box transcription factor family, FOXO1 has gained increasing attention from researchers in recent years. The transcription factor is a key regulator of cell 
A

AN3 CA

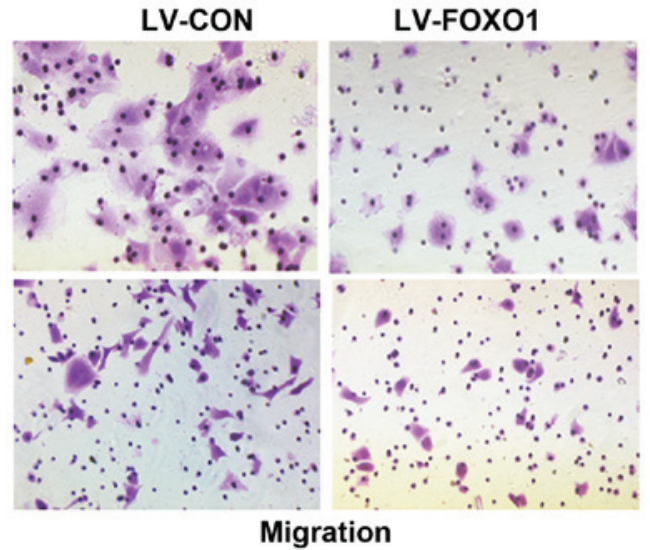

C

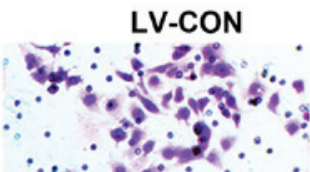

LV-FOXO1

Ishikawa

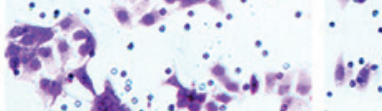

AN3 CA

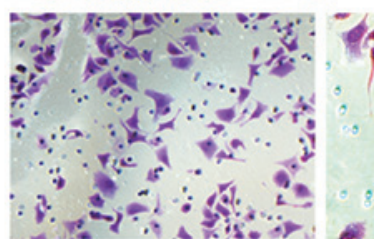

\section{Invasion}

B

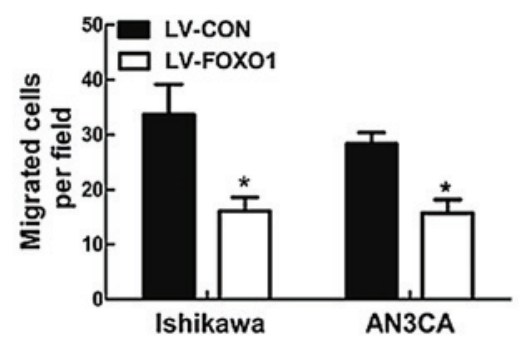

D

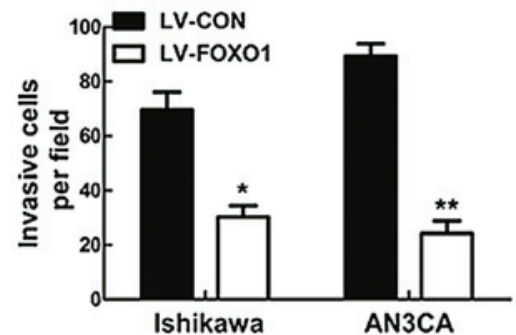

Figure 3. Stable overexpression of FOXO1 suppresses cell migration and invasion in vitro. Overexpression of FOXO1 inhibited cell migration of the (A) Ishikawa and (B) AN3 CA cells. Overexpression of FOXO1 inhibited cell invasion in the (C) Ishikawa and (D) AN3 CA cells. " $\mathrm{P}<0.05$ and ${ }^{* *} \mathrm{P}<0.01 \mathrm{vs.} \mathrm{the} \mathrm{LV-CON}$ group. FOXO1, forkhead transcription factor 1.

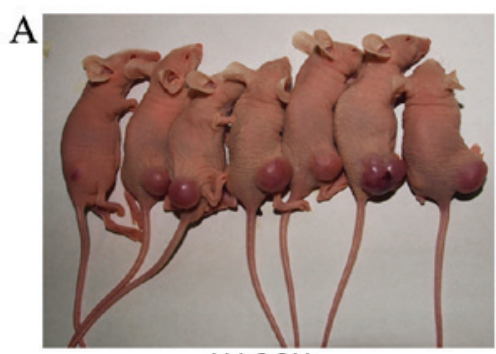

LV-CON

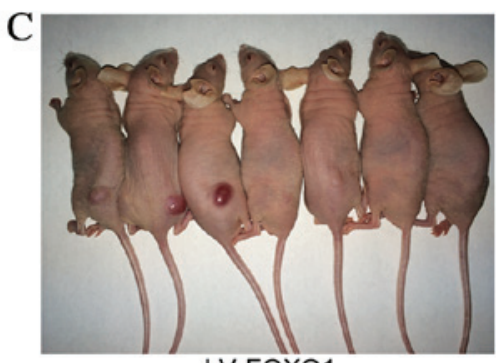

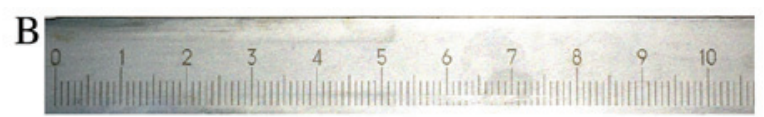

LV-CON

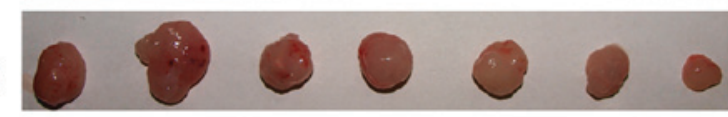

LV-FOXO1

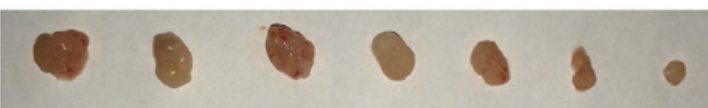

$\mathrm{D}$

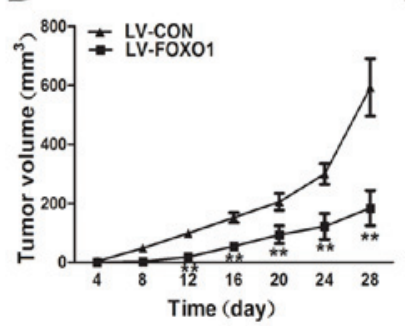

$\mathrm{E}$

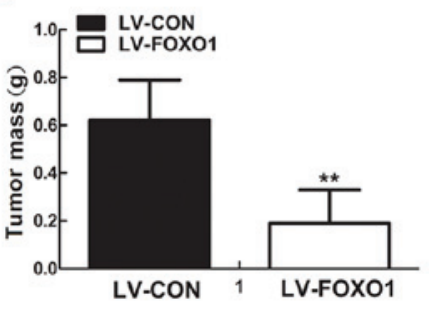

Figure 4. Stable overexpression of FOXO1 suppresses tumorigenesis in vivo in a xenograft model. (A) Stably transfected AN3 CA (LV-CON) were injected into the flank of nude mice. (B) Day 28 post-injection tumors were separated from the flank of nude mice. (C) Stably transfected AN3 CA (FOXO1-CON) cells were injected into the flank of nude mice. (D) Overexpression of FOXO1 inhibited tumor volume. (E) Overexpression of FOXO1 inhibited tumor weight. ${ }^{* *} \mathrm{P}<0.01$ vs. the LV-CON group. FOXO1, forkhead transcription factor 1.

fate, regulating cell differentiation, cell cycle arrest, apoptosis and defense responses against oxidative stress (20). Loss of FOXO1 expression may result in uncontrolled cell proliferation and lead to tumorigenesis, which has been reported in various forms of cancer, including ovarian cancer (21), prostate cancer $(22,23)$, lung cancer (24), breast cancer (25-27) and 
gastric cancer (28). Furthermore, downregulation of FOXO1 serves an important role in EC tumorigenesis $(15,29,30)$.

FOXO1 is a downstream target of the phosphatase and tensin homolog (PTEN)/phosphoinositide 3-kinase (PI3K)/Akt pathway (31). Downregulation and dysfunction of PTEN/PI3K/Akt signaling is a hallmark of EC (31). Research has demonstrated that a $\sim 55 \%$ decrease in PTEN expression may be observed in total endometrial lesions and $\sim 80 \%$ of PTEN is inactivated in EC cases (32). Low PTEN expression is considered to be an early event of EC $(33,34)$. Activation of the PTEN/PI3K/Akt pathway leads to the Akt-dependent phosphorylation of FOXO1, which subsequently promotes FOXO1 translocation from the nucleus to the cytoplasm, resulting in the inhibition of FOXO1 transcription activation (35). This process is important for cell apoptosis and differentiation (36). Ward et al (14) demonstrated that FOXO1 exhibited lower expression levels in EC samples compared with normal endometrium tissue. Loss of FOXO1 expression promotes uncontrolled EC cell proliferation. An additional study demonstrated that FOXO1 expression levels were high in HEC-1B cells and low in Ishikawa cells (15), which is consistent with the results of the present study. Furthermore, the present study identified that in vitro overexpression of FOXO1 was able to suppress the proliferative, colonigenic, migratory and invasive ability of Ishikawa and AN3 CA cells, and in vivo FOXO1 overexpression was able to suppress AN3 CA cell proliferation. These results were in line with previous studies investigating in EC $(14,15)$, supporting the notion that FOXO1 is a tumor suppressor in EC.

Obesity is strongly associated with EC (37). One meta-analysis reported that when body mass index increased per $5 \mathrm{~kg} / \mathrm{m}^{2}$, the risk of a woman developing EC increased by $59 \%$ (38). Ectopic lipid metabolism serves an important role in the formation of endometrial cancer (38).

SREBP1 coded by SREBP1, also known as adipocyte determination and differentiation dependent factor 1 , is a transcription factor that primarily regulates lipid homeostasis by targeting genes in cholesterol and fatty acid synthesis (16). Intracellular sterol level is able to control the function of SREBP1. When sterol decreases within the cell, inactive precursors of SREBP1 transport to the Golgi apparatus where they are cleaved and become active; these are then released into the nucleus where they target genes involved in cholesterol biosynthesis (39). By contrast, when the level of sterol is high, SREBP1 remains inactive, thus maintaining a balance between sterol level and FA metabolism $(39,40)$. Several studies have demonstrated that high SREBP1 expression may result in tumor formation, including prostate (17), breast (18) and colon (41) cancer. In addition, a previous study reported that SREBP1 was overexpressed in EC and resulted in tumorigenesis (3). And another research of my group found that SIRT1 can regulate the lipogenesis by targeting the expression of SREBP1 in EC (42).

FOXO1 and SREBP1 are important in the lipogenesis and tumorigenesis of EC, and are all targets of insulin; thus, the present study speculated that there may be some relevance. Western blot analysis was performed and the results demonstrated that the protein level of SREBP1 in Ishikawa and AN3 CA transduced with lentiviruses containing FOXO1 overexpression vectors was lower than the control group. A previous study reported that FOXO1 is able to directly repress SREBP-1 expression in hepatic lipogenesis (43). In addition, the present study supported the hypothesis that increased FOXO1 expression decreases the level of SREBP1.

In conclusion, the present study demonstrated that FOXO1 is important in EC progression. High expression of FOXO1 is able to inhibit the capacity of EC proliferation in vitro and in vivo, in addition to inhibiting migration and invasion in vitro via SREBP1. This may possibly identify novel therapeutic target in EC, with further studies required to clarify the molecular mechanisms by which FOXO1 suppress SREBP1 expression in EC.

\section{Acknowledgements}

The present study was funded by the National Natural Science Foundation of China (nos. 81372808 and 81173614), the Technology Development planning of Shandong (no. 2012G0021823) and the Science and Technology Developing Planning of Jinan (no. 201303035).

\section{References}

1. Siegel RL, Miller KD and Jemal A: Cancer statistics, 2015. CA Cancer J Clin 65: 5-29, 2015.

2. Siegel RL, Miller KD and Jemal A: Cancer statistics, 2016. CA Cancer J Clin 66: 7-30, 2016.

3. Li W, Tai Y, Zhou J, Gu W, Bai Z, Zhou T, Zhong Z, McCue PA, Sang N, Ji JY, et al: Repression of endometrial tumor growth by targeting SREBP1 and lipogenesis. Cell Cycle 11: 2348-2358, 2012.

4. Swinnen JV, Brusselmans K and Verhoeven G: Increased lipogenesis in cancer cells: New players, novel targets. Curr Opin Clin Nutr Metab Care 9: 358-365, 2006

5. Modesitt SC, Hsu JY, Chowbina SR, Lawrence RT and Hoehn KL: Not all fat is equal: Differential gene expression and potential therapeutic targets in subcutaneous adipose, visceral adipose, and endometrium of obese women with and without endometrial cancer. Int J Gynecol Cancer 22: 732-741, 2012.

6. Zhang W, Patil S, Chauhan B, Guo S, Powell DR, Le J, Klotsas A, Matika R, Xiao X, Franks R, et al: FoxO1 regulates multiple metabolic pathways in the liver: Effects on gluconeogenic, glycolytic, and lipogenic gene expression. J Biol Chem 281: 10105-10117, 2006.

7. Kousteni S: FoxO1, the transcriptional chief of staff of energy metabolism. Bone 50: 437-443, 2012.

8. Ho KK, Myatt SS and Lam EW: Many forks in the path: Cycling with FoxO. Oncogene 27: 2300-2311, 2008.

9. Chen C, Xu T, Zhou J, Yan Y, Li W, Yu H, Hu G, Ding X, Chen J and Lu Y: High cytoplasmic FOXO1 and pFOXO1 expression in astrocytomas are associated with worse surgical outcome. PLoS One 8: e69260, 2013.

10. Berry E, Hardt JL, Clardy J, Lurain JR and Kim JJ: Induction of apoptosis in endometrial cancer cells by psammaplysene A involves FOXO1. Gynecol Oncol 112: 331-336, 2009.

11. Greer EL and Brunet A: FOXO transcription factors at the interface between longevity and tumor suppression. Oncogene 24: 7410-7425, 2005.

12. Lettieri Barbato D, Aquilano K and Ciriolo MR: FoxO1 at the nexus between fat catabolism and longevity pathways. Biochim Biophys Acta: 1555-1560, 2014.

13. Pavlidou A and Vlahos NF: Molecular alterations of PI3K/Akt/mTOR pathway: A therapeutic target in endometrial cancer. ScientificWorld Journal: 709736, 2014.

14. Ward EC, Hoekstra AV, Blok LJ, Hanifi-Moghaddam P, Lurain JR, Singh DK, Buttin BM, Schink JC and Kim JJ: The regulation and function of the forkhead transcription factor, Forkhead box O1, is dependent on the progesterone receptor in endometrial carcinoma. Endocrinology 149: 1942-1950, 2008.

15. Goto T, Takano M, Albergaria A, Briese J,PomeranzKM, Cloke B, Fusi L, Feroze-Zaidi F, Maywald N, Sajin M, et al: Mechanism and functional consequences of loss of FOXO1 expression in endometrioid endometrial cancer cells. Oncogene 27: 9-19, 2008. 
16. Chen G, Liang G, Ou J, Goldstein JL and Brown MS: Central role for liver X receptor in insulin-mediated activation of Srebp-1c transcription and stimulation of fatty acid synthesis in liver. Proc Natl Acad Sci USA 101: 11245-11250, 2004.

17. Heemers H, Maes B, Foufelle F, Heyns W, Verhoeven G and Swinnen JV: Androgens stimulate lipogenic gene expression in prostate cancer cells by activation of the sterol regulatory element-binding protein cleavage activating protein/sterol regulatory element-binding protein pathway. Mol Endocrinol 15: 1817-1828, 2001.

18. Yang Y, Morin PJ, Han WF, Chen T, Bornman DM, Gabrielson EW and Pizer ES: Regulation of fatty acid synthase expression in breast cancer by sterol regulatory element binding protein-1c. Exp Cell Res 282: 132-137, 2003.

19. Sun Y, He W, Luo M, Zhou Y, Chang G, Ren W, Wu K, Li X, Shen J, Zhao X and Hu Y: SREBP1 regulates tumorigenesis and prognosis of pancreatic cancer through targeting lipid metabolism. Tumour Biol 36: 4133-4141, 2015.

20. Xiao E and Graves DT: Impact of diabetes on the protective role of FOXO1 in wound healing. J Dent Res 94: 1025-1026, 2015.

21. Wang J, Yang H, Li W, Xu H, Yang X and Gan L: Thioredoxin 1 upregulates FOXO1 transcriptional activity in drug resistance in ovarian cancer cells. Biochim Biophys Acta 1852: 395-405, 2015.

22. Zhao Y, Tindall DJ and Huang H: Modulation of androgen receptor by FOXA1 and FOXO1 factors in prostate cancer. Int J Biol Sci 10: 614-619, 2014.

23. Yu JJ, Wu YX, Zhao FJ and Xia SJ: miR-96 promotes cell proliferation and clonogenicity by down-regulating of FOXO1 in prostate cancer cells. Med Oncol 31: 910, 2014

24. Zhao JG, Ren KM and Tang J: Zinc finger protein ZBTB20 promotes cell proliferation in non-small cell lung cancer through repression of FoxO1. FEBS Lett 588: 4536-4542, 2014.

25. Yu F, Jin L, Yang G, Ji L, Wang F and Lu Z: Post-transcriptional repression of FOXO1 by QKI results in low levels of FOXO1 expression in breast cancer cells. Oncol. Rep 31: 1459-1465, 2014

26. Yang J, Li T, Gao C, Lv X, Liu K, Song H, Xing Y and Xi T: FOXO1 3'UTR functions as a ceRNA in repressing the metastases of breast cancer cells via regulating miRNA activity. FEBS Lett 588: 3218-3224, 2014

27. Wang X, Lin C, Zhao X, Liu A, Zhu J, Li X and Song L: Acylglycerol kinase promotes cell proliferation and tumorigenicity in breast cancer via suppression of the FOXO1 transcription factor. Mol Cancer 13: 106, 2014.

28. Yu DA, Yoon J, Ko YS, Park J, Kim SY, Kim MA, Kim JH, Jung J, Cheon Y, Lee HS, et al: Forkhead transcription factor FOXO1 inhibits nuclear factor- $\kappa \mathrm{B}$ in gastric cancer. APMIS 122 : 848-855, 2014

29. Myatt SS, Wang J, Monteiro LJ, Christian M, Ho KK, Fusi L, Dina RE, Brosens JJ, Ghaem-Maghami S and Lam EW Definition of microRNAs that repress expression of the tumor suppressor gene FOXO1 in endometrial cancer. Cancer Res 70: 367-377, 2010.
30. Korani M, Fallah S, Tehranian A, Nourbakhsh M, Samadikuchaksaraei A, Pour MS and Maleki J: The evaluation of the FOXO1, KLF9 and YT521 genes expression in human endometrial cancer. Clin Lab 59: 483-489, 2013.

31. Bansal N, Yendluri V and Wenham RM: The molecular biology of endometrial cancers, and the implications for pathogenesis, classification and targeted therapies. Cancer Control 16: 8-13, 2009.

32. Ali IU: Gatekeeper for endometrium: The PTEN tumor suppressor gene. J Natl Cancer Inst 92: 861-863, 2000.

33. Cully M, You H, Levine AJ and Mak TW: Beyond PTEN mutations: The PI3K pathway as an integrator of multiple inputs during tumorigenesis. Nat Rev Cancer 6: 184-192, 2006.

34. Mutter GL, Lin MC, Fitzgerald JT, Kum JB, Baak JP, Lees JA, Weng LP and Eng C: Altered PTEN expression as a diagnostic marker for the earliest endometrial precancers. J Natl Cancer Inst 92: 924-930, 2000.

35. Myatt SS and Lam EW: The emerging roles of forkhead box (Fox) proteins in cancer. Nat Rev Cancer 7: 847-859, 2007.

36. Modur V, Nagarajan R, Evers BM and Milbrandt J: FOXO proteins regulate tumor necrosis factor-related apoptosis inducing ligand expression. Implications for PTEN mutation in prostate cancer. J Biol Chem 277: 47928-47937, 2002.

37. Schmandt RE, Iglesias DA, Co NN and Lu KH: Understanding obesity and endometrial cancer risk: Opportunities for prevention. Am J Obstet Gynecol 205: 518-525, 2011.

38. Renehan AG, Tyson M, Egger M, Heller RF and Zwahlen M: Body-mass index and incidence of cancer: A systematic review and meta-analysis of prospective observational studies. Lancet 371: 569-578, 2008.

39. Shimano H: Sterol regulatory element-binding proteins (SREBPs): Transcriptional regulators of lipid synthetic genes. Prog Lipid Res 40: 439-452, 2001.

40. Horton JD and Shimomura I: Sterol regulatory element-binding proteins: Activators of cholesterol and fatty acid biosynthesis Curr Opin Lipidol 10: 143-150, 1999.

41. Schønberg SA, Lundemo AG, Fladvad T, Holmgren K, Bremseth H, Nilsen A, Gederaas O, Tvedt KE, Egeberg KW and Krokan HE: Closely related colon cancer cell lines display different sensitivity to polyunsaturated fatty acids, accumulate different lipid classes and downregulate sterol regulatory element-binding protein 1. FEBS J 273: 2749-2765, 2006.

42. Lin L, Zheng X, Qiu C, Dongol S, Lv Q, Jiang J, Kong B and Wang C: SIRT1 promotes endometrial tumor growth by targeting SREBP1 and lipogenesis. Oncol Rep 32: 2831-2835, 2014.

43. Deng X, Zhang W, O-Sullivan I, Williams JB, Dong Q, Park EA, Raghow R, Unterman TG and Elam MB: FoxO1 inhibits sterol regulatory element-binding protein-1c (SREBP-1c) gene expression via transcription factors Sp1 and SREBP-1c. J Biol Chem 287: 20132-20143, 2012. 